\title{
Design of Android-Based Remote Terminal System for RGV
}

\author{
Shang Qinghua*, Zhao Xinyue, Zhang Helong and Wang Su \\ Measurement and Control Technology and Application laboratory of \\ Heilongjiang Province, Harbin University of Science and Technology, Harbin \\ 150080, China \\ $568382891 @ q q . c o m$
}

\begin{abstract}
This paper proposes a new method that utilized Android mobile phone to control the $R G V$ (Rail Guided Vehicle)running through WIFI network to overcome the disadvantages of the traditional remote control can only control one-way communication for $R G V$ in the Logistics and warehousing and realize two-way communication between the RGV and remote controller. The system is based on Android platform, and established a remote terminal system by all Android support resources. In this paper, it described the structure, function, software flow of the RGV remote terminal system and communication protocol between Android client and the RGV. The design is based on the Modbus TCP / IP protocol, to achieve a two-way communication between Android phone and the RGV.
\end{abstract}

Keywords: RGV; Android; Communication; Modbus; Terminal System

\section{Introduction}

RGV shelves is a type of dense storage devices which has the advantages of simple structure, high safety, high efficiency and space utilization. The large cargo throughput and operations frequently such as home appliances, the tobacco and food with high demand for goods is the biggest source. RGV is a car that is used for warehousing and logistics operations between the shelves. RGV operation is simple, precise job security, access to goods with a shorter time, it can not only improve the work efficiency when used, but also connect with other logistics systems automatically, overcoming the shortcomings of manual handling of goods[1]. Now generally used to control the RGV run is an infrared remote control or radio remote control [2]. It has a lot of disadvantages, controlling RGV with the remote control are one-way communication, it can only control the RGV run but the running status and fault information of the RGV can not be transmitted to the remote control terminal system, it also cannot show the current running track of the RGV and the power of the battery inside. With the rapid development of smart phones, the popularity of WiFi technology and the more perfect Android system [3], using Android mobile phone to develop the program to control RGV can be a good solution to the above problems, overcame the disadvantages of traditional remote controller can only control the RGV forward, back, arm up, arm down and stop these five operating methods. Android mobile phone via WiFi technology can communicate with the RGV so that it can receive the inventory of input or output of the goods, and it can carry out the management of input operation or output operation, it also can show clearly the running track and the battery power of the RGV, the failure of RGV running process will be fed timely to the Android client. Any Android mobile phone that install the RGV remote terminal APP can realize the operation of controlling the RGV run. 


\section{System Overall Structure}

RGV remote terminal system is composed of Android mobile client and RGV control system. As shown in Figure 1.
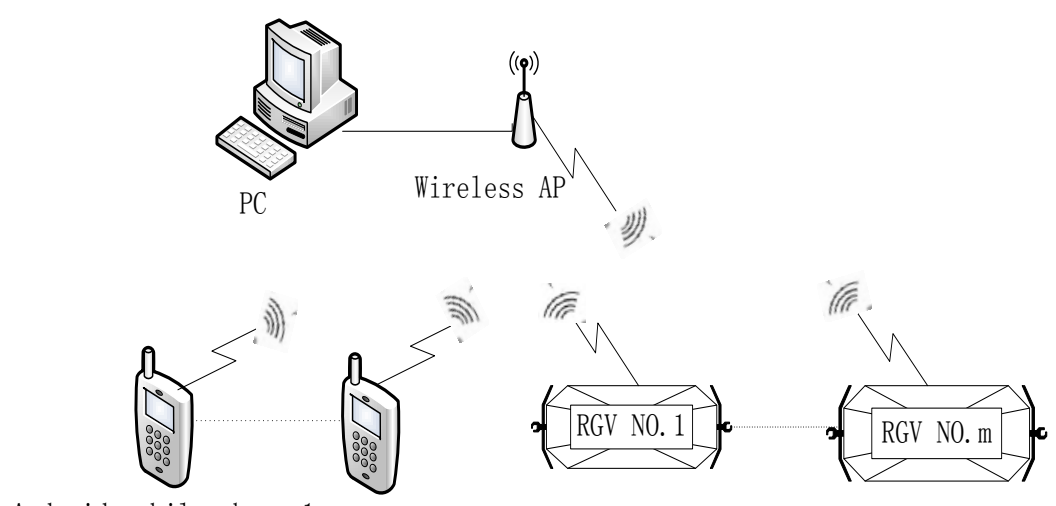

Android mobile phone 1

Android mobile phone $n$

Figure 1. Remote Terminal System Configuration Diagram

The main role of the PC is to achieve the management of goods. PC will send management information to the Android mobile phone when carrying out the input or output operation, so that the operators could operate the RGV run to complete the input / output of the goods. The WiFi signal in the RGV's shelf area is provided by the wireless AP. RGV through the internal programmable controller PLC to communicate with the Android mobile client, controlling the RGV run and send running status information or failure information to the Android mobile client.

\section{Software Function}

Modular design method is applied in the software of the remote terminal system, which makes it more convenient for programming, maintaining and upgrading. The functional diagram of the software system is shown in Figure 2. 


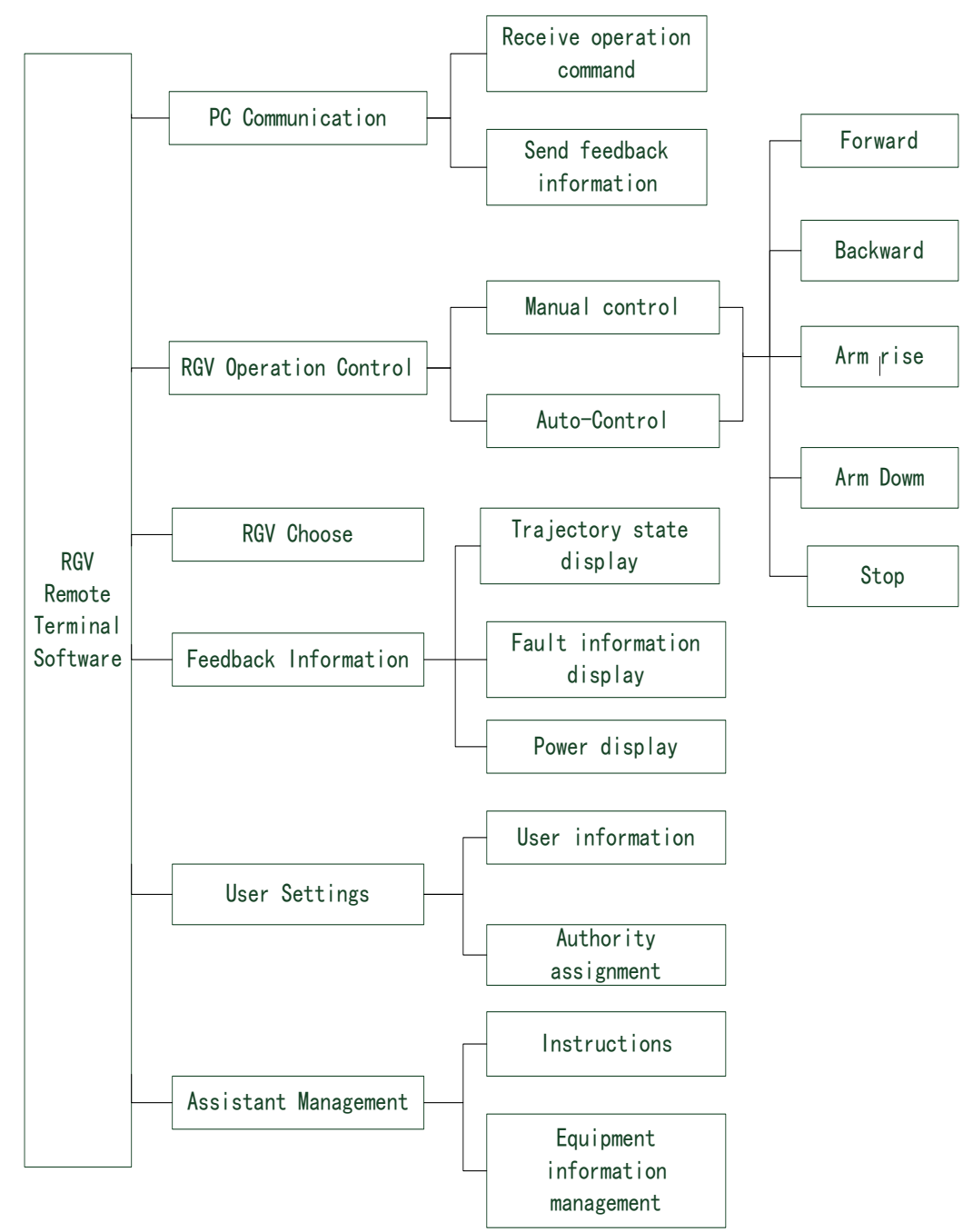

Figure 2. Software System Functional Block Diagram

1). Communication between $P C$ and the remote terminal system

PC is mainly for cargo into / out of warehouse management and send information of into / out of warehouse to the Android mobile phone client. According to the information, operators operating the RGV to run to transport the goods to the specific shelf or remove the goods from the shelves. PC will receive the feedback information from the Android mobile client.

2). RGV operation control

The control function of the RGV includes automatic control and manual control. Which also includes the RGV forward, back, arm up, arm down and stop these five functions. Selecting the automatic control, the RGV will be in accordance with the specific model to work. If select manual operation, the operator control the operation of the RGV through the key on the mobile phone screen of the Android mobile phone client.

3). Choosing the RGV

There are many RGV's in the warehouse and logistics, after the operators receive the information of the goods of into / out of the warehouse from the PC, choosing the appropriate RGV to control it to complete the management of into/ out of the warehouse.

4). Feedback information management

The feedback information management includes the movement track of the RGV and the display of the fault information. The interface of the RGV remote terminal can show clearly the running track and the battery power of the RGV. The RGV will be put the fault 
information feedback to the Android mobile phone client, to remind the operator to deal with it.

5). User permission setting

It provided high quality authority definitions, according to the privileges to control the operational range of the operators.

6). Aid management

Users can read the operating instructions of this system to record the variety of information when the device running, for example, system feedback information, operating system information, alarm untreated and so on.

\section{Communication Protocol}

Modbus protocol is one of the most popular protocols in the industrial protocols used today. It is simple, inexpensive, versatile and easy to use. The Modbus protocol defined a generic message structure that describes a process that the controller requests to access other devices, including how to respond to the requests from other devices, and how to detect errors and records, and to develop a public format for message domains and contents. When communicating through this network, each controller has a unique address, and to recognize the messages sent by the address. At last, deciding to produce what kind of action. If it need to respond, then the controller will generate feedback information and make a response through this protocol [4]. In this system, the communication between the Android client and the RGV uses the Modbus TCP/IP protocol. The TCP/IP Modbus protocol data frame format is shown in Figure 3 [5].

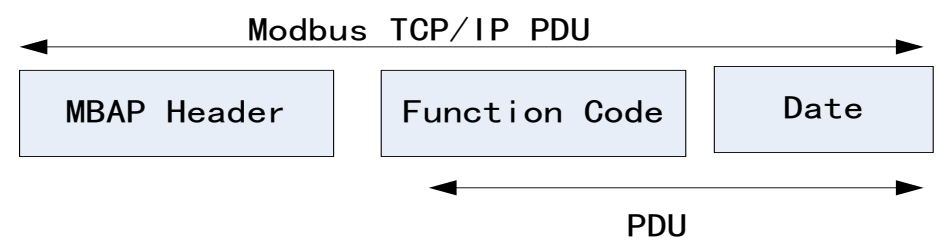

Figure 3. Modbus TCP/IP Data Frame Structure Diagram

The MBAP header is 7 bytes, including the transaction identifier, the protocol identifier, the length, and the cell identifier. The Modbus TCP/IP data frame is applied to the communication that between the Android client and the RGV control terminal. The required function code is shown in Table 1.

Table 1. Modbus Function Code

\begin{tabular}{c|c|c|c|c|c|c|c|c}
\hline $\begin{array}{l}\text { Function } \\
\text { code }\end{array}$ & 00 & 01 & 02 & 03 & 04 & 05 & 06 & 07 \\
\hline Name & Stop & Forward & Backward & Rise & Down & Power & Orbit & Warning \\
\hline
\end{tabular}

The function code in the RGV remote terminal system, 00 04, represent 5 major operations of the RGV that controlled by the Android mobile client. There is no data segment behind of it. 05, represents the battery information of the RGV, and the data behind of the data segment is the percentage of the battery information. 06 is the movement track information of the RGV, the data in the back of the data segment indicates the position information that fed by the controller of the RGV. 07, represents the fault warning, the data in the back of the data segment show the name of the fault that may occur. Fault data code as shown in Table 2. 
Table 2. Fault Data Code

\begin{tabular}{|l|l|}
\hline $\begin{array}{l}\text { Fault } \\
\text { data } \\
\text { code }\end{array}$ & Name \\
\hline 01 & Walking motor overheating \\
\hline 02 & Lifting motor overheating \\
\hline 03 & POST abnormal \\
\hline 04 & Lifting action Timeout \\
\hline 05 & $\begin{array}{l}\text { Return to the starting position } \\
\text { timeout }\end{array}$ \\
\hline 06 & Battery low voltage alarm \\
\hline 07 & Walking motion Timeout \\
\hline 08 & Goods placed abnormalities \\
\hline
\end{tabular}

In the running process of the RGV, when the alarm display appears on the Android client, the operator should stop the operation of the RGV, according to the fault code that is fed back, determining what kind of fault happened with the RGV, detecting the RGV, processing fault timely, ensure the RGV system efficient, reasonable operation, complete all kinds of warehouse operations.

\section{Software Process and Software Interface}

Android system architecture includes five parts, namely, application, Application Framework, Development Library, Android running environment and the Linux kernel system [6-7]. Using Eclipse as the development environment, ADT as the development of plugins [8]. The encode realization is based on Android SDK, JAVA as a programming language, while the software uses the threading technology that be provided by Android to ensure the fluency of the interface and the operation. Software development is mainly to complete the communication connection between mobile client and the RGV control terminal.

When the Android client communicate with the RGV control system, after the firstly connected wireless WiFi [9], see tips for a successful connection, then the operator can input the correct user name and password, PC will send the into / out of the warehouse information to the Android client, when the operators receive the information from the PC and then choose the appropriate RGV to work. The main flow chart of the operating software is shown in Figure 4. 


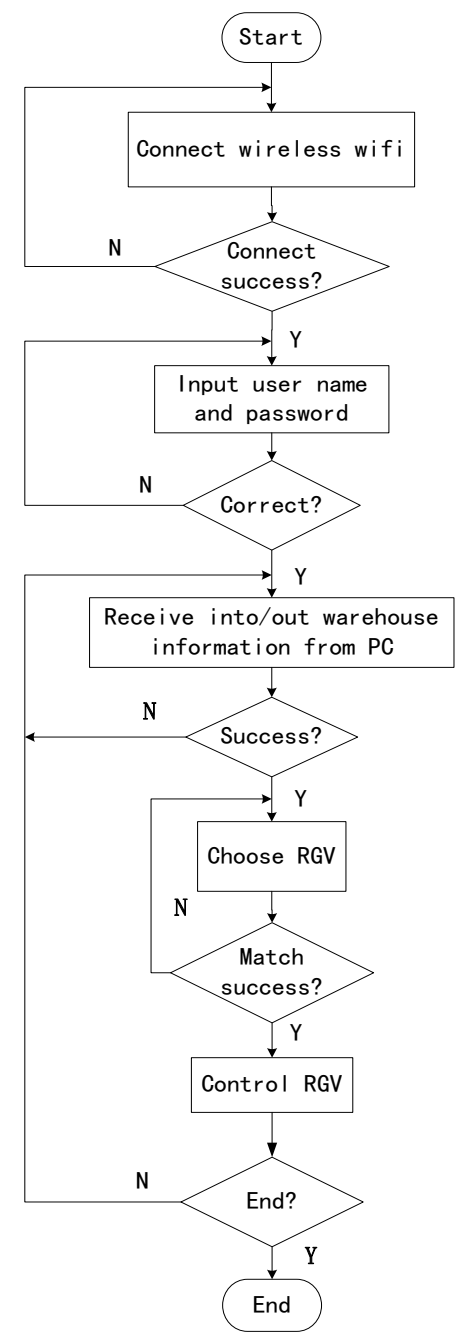

Figure 4. The Functional Block Principle Diagram of the Ventilation Control Part

When the operator choose to manually control the RGV, the RGV control interface which on the Android mobile client is shown in Figure 5, first determine whether the key, if there is to be the key then recognition, the functions of the key including the rise, fall, forward, backward and stop ,determining what action to the RGV, then operator began to send the function codes to RGV control terminal, after receiving the message, the PLC controller in the RGV will control the RGV to complete the operation of the goods that into/out of the warehouse [10]. When the RGV in case of failure during operation, the control terminal will immediately send the fault information to the Android client, the operator can be based on the fault message on the software interface to make a response.

The main process of the automatic control of the RGV is the same as the manual operation. But the button operation, the RGV will be run in particular pattern to complete the operation of the goods into/out the warehouse. 


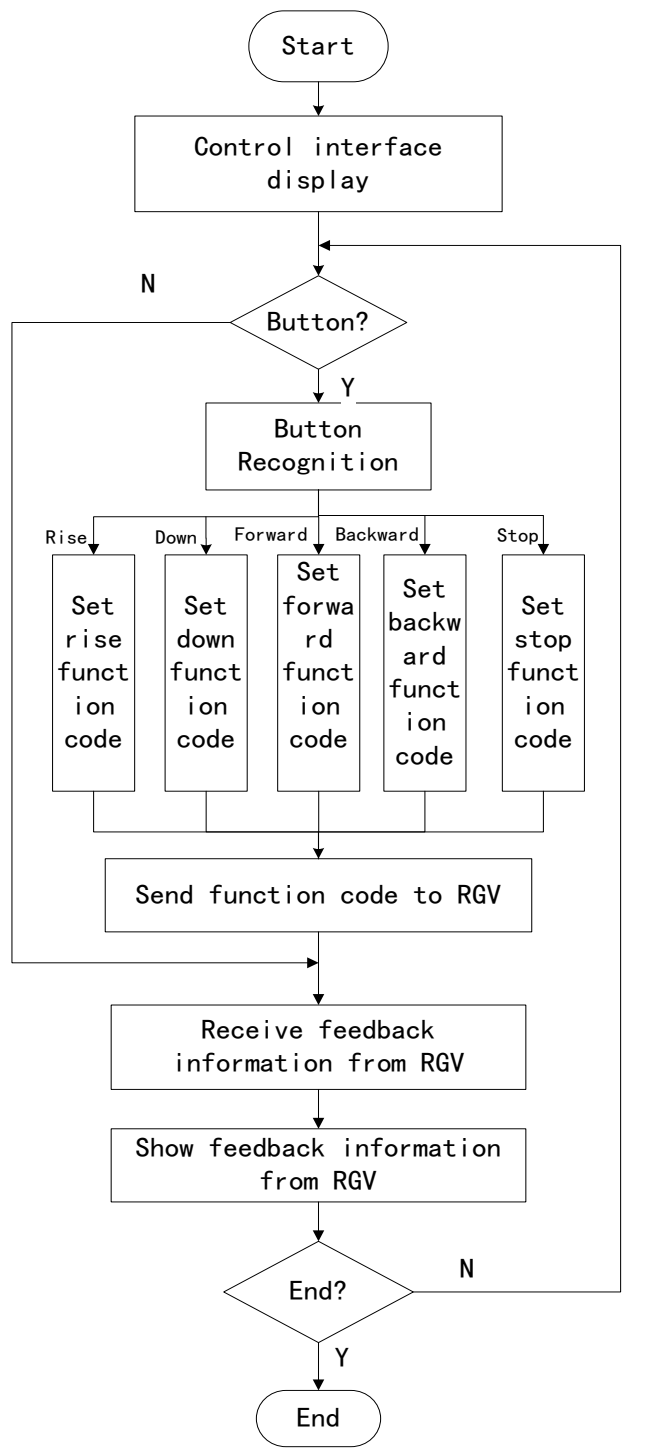

\section{Figure 5. Manual Operation Control Software Flow Chart for RGV}

The software design of the remote terminal system of RGV has the characteristics of simple interface and convenient operation. Client design is mainly related to the user interface, socket communications, threads and SQLite database design and use. Many components are applied to the application programs, such as activity Group, Service, activity, progress bar, handle list view and so on, each component has completed it's corresponding tasks, using different layout method to complete the user interface display.

Figure 6 is the software interface layout of the part of the Android client, the main role of the software interface layout is the integrated management, providing the interface of each module, clicking the corresponding button to control the corresponding function, the development language is Java. Figure 6 (a) is the Android client to visit the software interface, Figure 6 (b) is a diagram that choosing the appropriate RGV to run after the landing is completed. Figure 6 (c) is a diagram that the operator control the RGV to complete the into / out of the warehouse management operation. Figure 6 (d) is a diagram that the running track of the RGV. 


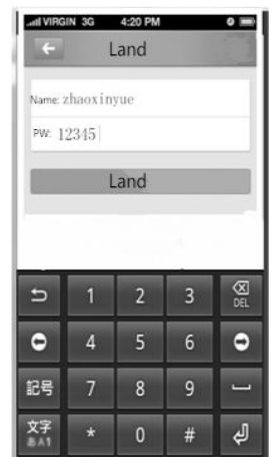

(a) Login interface Diagram

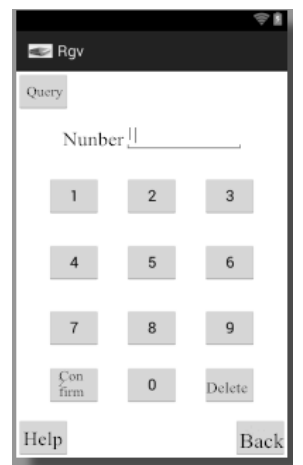

(b) Choose RGV

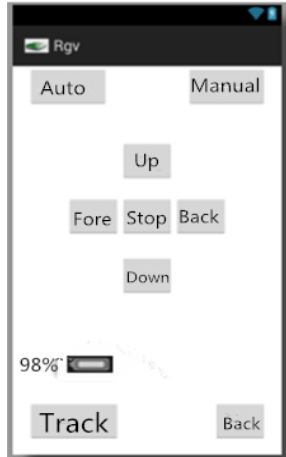

(c) Control RGV

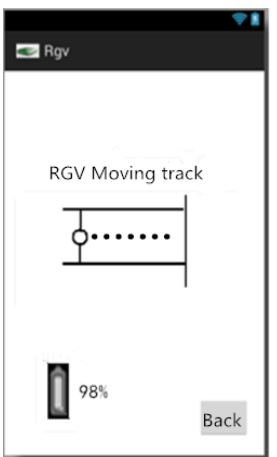

(d) RGV Moving Interface Diagram Interface Diagram Track Diagram

Figure 6. Software Interface Diagram

\section{Conclusion}

RGV remote terminal system has completely changed the status that control the RGV run through the traditional remote control, realizing the real meaning two-way wireless communication between the android client and the RGV. So the control operation is more flexible, convenient, low cost, saving time, improving greatly the work efficiency of the warehouse logistics system. Through the practical application shows the system can reduce the production cost, reduce production accidents and improve the economic efficiency and has a good application prospect.

\section{References}

[1] X. Jingjing and L. Xiaojuan, "RGV System and Its Application”, Logistics technology and Application, no. 2, (2012), pp. 69-72.

[2] X. Yanggao, "RGV Control System Solution", Logistics technology and Application. no. 7, (2013).

[3] R. Runun, "Application software development based on Android operating system", Xi'an Electronic and Science University, (2013).

[4] M. Hua and W. Pengda, "Implementation of Communication between PIC Signal Chip Professor and Touch-screen Based on Modbus Protocol", no. 10, (2009), pp. 58-75.

[5] S. Liping, H. Guiming and C. Mingbang, "Industrial control communication based on MODBUS/TCP protocol", Computer application, no. 25, (2005), pp. 29-31.

[6] L. Xinghua, "Android development classic case", Tsinghua University press, (2012), pp. 440-445.

[7] L. Gang, "Crazy Android notes", Beijing Electronics Industry Press, (2013), pp. 580.

[8] Google. Android SDK Documentation [EB/OL]. [2011-05-18]. http: //code. Google.com/ android/ documentation.html.

[9] L. Jiajie and Z. Mingfu, "Research on communication of real time monitoring system based on Android terminal", Journal of Changzhou Institute of Technology, no. 12, (2015), pp. 7-10.

[10] L. Dongmei and Y. Xiaodai, "Control and Application of Shuttle Vehicle in Automatic Material Handling System”, Tobacco Science and Technology, no. 10, (2002), pp. 23-25. 